\title{
Gelfand-Kirillov dimension of differential difference algebras
}

\author{
Yang Zhang and Xiangui Zhao
}

\begin{abstract}
Differential difference algebras, introduced by Mansfield and Szanto, arose naturally from differential difference equations. In this paper, we investigate the Gelfand-Kirillov dimension of differential difference algebras. We give a lower bound of the Gelfand-Kirillov dimension of a differential difference algebra and a sufficient condition under which the lower bound is reached; we also find an upper bound of this Gelfand-Kirillov dimension under some specific conditions and construct an example to show that this upper bound cannot be sharpened any further.
\end{abstract}

\section{Introduction}

Differential difference algebras (Definition 2.1) arose naturally from differential difference equations $[\mathbf{2}, \mathbf{1 0}]$. The class of differential difference algebras contains several well-known classes of noncommutative algebras, for example, commutative polynomial algebras, quantum planes, and skew polynomial algebras of derivation (or automorphism) type. Roughly speaking, a differential difference algebra is a noncommutative polynomial ring (over an algebra) with two sets $D$ and $S$ of indeterminates, where $D$ originally stands for differential operators and $S$ originally stands for shift operators (the difference operators can be derived from $S$ ). Operators in $D$ (S, respectively) commute with each other, but not with those in $S$ ( $D$, respectively). The exact definition is given in $\S 2$.

Let $k$ be a field and $A$ be a unital associative $k$-algebra. The Gelfand-Kirillov dimension of $A$ is defined as

$$
\operatorname{GKdim}(A)=\sup _{V} \varlimsup_{n \rightarrow \infty} \log _{n} \operatorname{dim}_{k}\left(V^{n}\right)
$$

where the supremum is taken over all finite-dimensional subspaces $V$ of $A$. The GelfandKirillov dimension is a very useful and powerful tool for investigating noncommutative algebras. Basic properties of Gelfand-Kirillov dimension can be found in [7].

There have been a number of results concerning Gelfand-Kirillov dimensions of algebras with derivations and/or automorphisms, for example, the Gelfand-Kirillov dimension of Ore extensions of derivation type [9], of Ore extensions of automorphism type [3], of PoincaréBirkhoff-Witt (PBW) extensions [11], and of skew polynomial extensions [13].

Assume that $R$ is a unital associative $k$-algebra. Let $\sigma$ be an automorphism of $R, \delta$ be a $\sigma$-derivation of $R$ and $A=R[x ; \sigma, \delta]$ be an Ore extension over $R$. It was shown in [3] that

$$
\operatorname{GKdim}(A) \geqslant \operatorname{GKdim}(R)+1 ;
$$

the inequality becomes an equality provided that the following condition holds:

(*) each finite-dimensional subspace $U$ of $R$ is contained in a finite-dimensional subspace $V$ such that $\sigma(V) \subseteq V$ and $\delta(V) \subseteq V^{p}$ for some $p \geqslant 1$.

Received 6 December 2013; revised 11 April 2014.

2010 Mathematics Subject Classification 16P90, 16S36 (primary).

This work was supported in part by the National Sciences and Engineering Research Council of Canada. The second named author was additionally supported by the Manitoba Graduate Scholarship.

Corresponding author: Xiangui Zhao 
In this paper we investigate the Gelfand-Kirillov dimension of differential difference algebras. We show that inequality (1) of the Gelfand-Kirillov dimension of an Ore extension can be extended to differential difference algebras, that is, we get a lower bound of the GelfandKirillov dimensions of differential difference algebras. However, owing to the noncommutativity of indeterminates of differential difference algebras, even under conditions similar to $(*)$, the equality does not hold for differential difference algebras in general. We find an upper bound for the Gelfand-Kirillov dimension of a differential difference algebra satisfying (an analogue of ) condition $(*)$, and give a sufficient condition under which the lower bound is reached. We also construct an example to show that the upper bound we obtain cannot be sharpened any further.

This paper is organized as follows. Definition and examples of differential difference algebras are given in $\S 2$. The Gelfand-Kirillov dimension of differential difference algebras is investigated in $\S 3$.

\section{Preliminaries}

Throughout this paper, we assume that $k$ is a field and all algebras are unital associative $k$ algebras. Denote the set of $k$-algebra automorphisms of algebra $A$ by $\operatorname{Aut}(A)$. If $\sigma \in \operatorname{Aut}(A)$, then a mapping $\delta$ on $A$ is called a $\sigma$-derivation provided that, for any $a, b \in A$ and $c \in k$, $\delta(c a+b)=c \delta(a)+\delta(b)$ and $\delta(a b)=\sigma(a) \delta(b)+\delta(a) b$. In particular, if $\sigma=\mathrm{id}$, then $\delta$ is called a derivation on $A$.

First we recall the definition of differential difference algebras, which was introduced by Mansfield and Szanto [10] with some discussions of Gröbner bases.

Definition 2.1 (cf. [10]). An algebra $A$ is called a differential difference algebra of type $(m, n), m, n \geqslant 1$, over a subalgebra $R \subseteq A$ if there exist elements $S_{1}, \ldots, S_{m}, D_{1}, \ldots, D_{n}$ in $A$ such that:

(i) the set $\left\{S_{1}^{\alpha_{1}} \ldots S_{m}^{\alpha_{m}} D_{1}^{\beta_{1}} \ldots D_{n}^{\beta_{n}}: \alpha_{i}, \beta_{j} \in \mathbb{N}, 1 \leqslant i \leqslant m, 1 \leqslant j \leqslant n\right\}$ forms a basis for $A$ as a free left $R$-module;

(ii) $D_{i} r=r D_{i}+\delta_{i}(r)$ for any $1 \leqslant i \leqslant n$ and $r \in R$, where $\delta_{i}$ is a derivation on $R$;

(iii) $S_{i} r=\sigma_{i}(r) S_{i}$ for any $1 \leqslant i \leqslant m$ and $r \in R$, where $\sigma_{i}$ is a $k$-algebra automorphism on the subalgebra $R\left[D_{1}, \ldots, D_{n}\right] \subseteq A$ such that $\left.\sigma_{i}\right|_{R} \in \operatorname{Aut}(R)$ and $\sigma_{i}\left(D_{j}\right)=\sum_{l=1}^{n} a_{i j l} D_{l}, \quad a_{i j l} \in R$;

(iv) $S_{i} S_{j}=S_{j} S_{i}, 1 \leqslant i, j \leqslant m ; D_{i^{\prime}} D_{j^{\prime}}=D_{j^{\prime}} D_{i^{\prime}}, 1 \leqslant i^{\prime}, j^{\prime} \leqslant n$;

(v) $D_{i} S_{j}=S_{j} \sigma_{j}\left(D_{i}\right), 1 \leqslant i \leqslant n, 1 \leqslant j \leqslant m$

(vi) for any $1 \leqslant i, j \leqslant n$ and $1 \leqslant i^{\prime}, j^{\prime} \leqslant m, \delta_{i} \circ \delta_{j}=\delta_{j} \circ \delta_{i}, \sigma_{i^{\prime}} \circ \sigma_{j^{\prime}}=\sigma_{j^{\prime}} \circ \sigma_{i^{\prime}}$.

REMARK 2.2. In the above definition, both subalgebras $R\left[D_{1}, \ldots, D_{n}\right]$ and $R\left[S_{1}, \ldots, S_{m}\right]$ of $A$ are iterated Ore extensions over $R$. But, in general $A$ is not an iterated Ore extension over $R$.

The class of differential difference algebras contains several other known classes of algebras, for example, commutative polynomial algebras, quantum planes, and skew polynomial algebras of derivation (or automorphism) type.

ExAmple 2.3. Let $0 \neq q \in k$ and $I_{q}$ be the two-sided ideal of the free associative algebra $k\langle x, y\rangle$ generated by the element $y x-q x y$. Then the quotient algebra

$$
k_{q}[x, y]=k\langle x, y\rangle / I_{q}
$$

is called a quantum plane $\left[\mathbf{6}\right.$, Chapter IV]. It is easy to see that $k_{q}[x, y]$ is a differential difference algebra of type $(1,1)$ over $k$.

The following example distinguishes differential difference algebras from algebras of solvable type [5], PBW extensions [1], and G-algebras [8]. 
EXAmple 2.4. Let $A$ be the $k$-algebra generated by $\left\{D_{1}, D_{2}, S_{1}\right\}$ with defining relations $\mathcal{R}=\left\{D_{2} D_{1}=D_{1} D_{2}, D_{1} S_{1}=S_{1} D_{2}, D_{2} S_{1}=S_{1} D_{1}\right\}$. Then it is easy to see that $A$ is a differential difference algebra of type $(1,2)$ over $k$. However, by the defining relations, $A$ is not an algebra of solvable type [5], PBW extensions [1], or G-algebras [8].

Let $\mathcal{D}=\left\{D_{1}, \ldots, D_{n}\right\}$ and $\mathcal{S}=\left\{S_{1}, \ldots, S_{m}\right\}$. If $A$ is a differential difference algebra over $R$ defined as Definition 2.1, we denote $A=R[\mathcal{S}, \mathcal{D} ; \sigma, \delta]$. For $\alpha=\left(\alpha_{1}, \ldots, \alpha_{m}\right) \in \mathbb{N}^{m}$ and $r \in R$, we simply write $\sigma^{\alpha}(r)=\sigma_{1}^{\alpha_{1}} \ldots \sigma_{m}^{\alpha_{m}}(r), \mathcal{D}^{\alpha}=D_{1}^{\alpha_{1}} \ldots D_{m}^{\alpha_{m}}$ and $|\alpha|=\alpha_{1}+\ldots+\alpha_{m}$. In particular, $D_{i}^{0}=1$, the identity of $R$. Similarly, we use notation $\delta^{\alpha}(r), \mathcal{S}^{\beta}\left(\beta \in \mathbb{N}^{n}\right)$, and so on. Then every element in $A$ can be written uniquely in the form: $\sum_{\alpha, \beta} r_{\alpha, \beta} \mathcal{S}^{\alpha} \mathcal{D}^{\beta}$, where $r_{\alpha, \beta} \in R$ and only finitely many $r_{\alpha, \beta}$ are nonzero.

The following example is taken from $[\mathbf{1 0}]$ with some modifications. This example shows where the differential difference algebras come from.

ExAmple 2.5. Let $M, n, p \in \mathbb{N}$ and $p \geqslant 1$. Consider the following system, which arises from the calculation of symmetries of discrete systems (cf. [4]),

$$
\begin{gathered}
u_{n+M+1}=\omega\left(n, u_{n}, u_{n+1}, \ldots, u_{n+M}\right) ; \\
\mathcal{D}_{j} F\left(n, u_{n}, u_{n+1}, \ldots, u_{n+M}\right)=0, \quad 1 \leqslant j \leqslant p,
\end{gathered}
$$

where $F$ is the unknown function and $w$ is a given function in the field $\mathbb{Q}\left(n, u_{n}, \ldots, u_{n+M}\right)$ of rational functions over the rational numbers $\mathbb{Q}$ in indeterminates $n, u_{n}, u_{n+1}, \ldots, u_{n+M}$, such that $\partial \omega / \partial u_{n} \neq 0$, and $\mathcal{D}_{j}: T \rightarrow T$ is a linear operator of the form

$$
\mathcal{D}_{j}=\sum_{\alpha=\left(\alpha_{0}, \ldots, \alpha_{M}\right) \in \mathbb{N}^{M}, \beta \in \mathbb{N}} c_{\alpha, \beta} \circ s^{\beta} \circ \frac{\partial^{\alpha_{0}+\ldots+\alpha_{M}}}{\partial u_{n}^{\alpha_{0}} \ldots \partial u_{n+M}^{\alpha_{M}}},
$$

where $T=\mathbb{Q}\left(n, u_{n+t}: t \in \mathbb{Z}\right), c_{\alpha, \beta} \in \mathbb{Q}\left(n, u_{n+t}: t \in \mathbb{Z}\right)$ are multiplication operators and only finitely many $c_{\alpha, \beta}$ are nonzero, and $s$ is the shift operator defined by $s(n)=n+1$ and $s\left(u_{n}\right)=u_{n+1}$.

A natural approach to deal with this system is to consider those operators $\mathcal{D}_{j}$ and $s$ as elements of the noncommutative algebra $A$ over $R$ generated by operator variables $\left\{S, D_{n}, \ldots, D_{n+M}\right\}$, where $S$ denotes the shift operator $s$ and $D_{n+t}$ denotes the differential operator $\partial / \partial u_{n+t}$ for $0 \leqslant t \leqslant M$, subject to the following commutation rules:

$$
\begin{aligned}
D_{n+t} \circ S & =S \circ D_{n+t-1}+\frac{\partial \omega}{\partial u_{n+t}} \circ S \circ D_{n+M}, \quad 1 \leqslant t \leqslant M ; \\
D_{n} \circ S & =\frac{\partial \omega}{\partial u_{n}} \circ S \circ D_{n+M} ; \\
D_{n+t} \circ D_{n+t^{\prime}} & =D_{n+t^{\prime}} \circ D_{n+t}, \quad 0 \leqslant t, t^{\prime} \leqslant M ; \\
S \circ r & =s(r) \circ S, \quad r \in R ; \\
D_{n+t} \circ r & =r \circ D_{n+t}+\frac{\partial r}{\partial u_{n+t}}, \quad r \in R, 0 \leqslant t \leqslant M .
\end{aligned}
$$

Then $A=\mathbb{Q}\left(n, u_{n+t}: t \in \mathbb{Z}\right)[S, \mathcal{D} ; \sigma, \delta]$ is a differential difference algebra of type $(1, M+1)$ over $\mathbb{Q}\left(n, u_{n+t}: t \in \mathbb{Z}\right)$, where $\delta_{i}=\partial / \partial u_{i}$ for $n \leqslant i \leqslant n+M$ and $\left.\sigma\right|_{R}=s, \sigma\left(D_{n}\right)=$ $s^{-1}\left(\partial \omega / \partial u_{n}\right) D_{n+M}, \sigma\left(D_{n+t}\right)=D_{n+t-1}+s^{-1}\left(\partial \omega / \partial u_{n+t}\right) D_{n+M}$ for $1 \leqslant t \leqslant M$.

Note that the difference operator $\Delta$, defined by $\Delta\left(u_{i}\right)=u_{i+1}-u_{i}$ for $i \in \mathbb{Z}$, can be derived from the shift operator: $\Delta=S-$ id. So the differential difference algebra in the above example actually involves both differential and difference operators. 


\section{Gelfand-Kirillov dimension of differential difference algebras}

In this section, we consider the Gelfand-Kirillov dimension of differential difference algebras.

We first fix some notation. Let $\operatorname{GKdim}(A)$ denote the Gelfand-Kirillov dimension of an algebra $A, \operatorname{dim}(V)$ denote the dimension of a $k$-vector space $V$, and $\operatorname{card}(T)$ denote the cardinality of a set $T$. Recall that, for $r, s \in \mathbb{N}$, the binomial coefficient $\left(\begin{array}{l}r \\ s\end{array}\right)=r(r-1) \ldots(r-s+1) / s(s-1) \ldots 1$ if $0 \leqslant s \leqslant r ;$ and $\left(\begin{array}{l}r \\ s\end{array}\right)=0$ if $s<0$ or $s>r$.

The Gelfand-Kirillov dimension of an Ore extension has been discussed in [3].

Proposition 3.1 ([3, Corollary 2.4], cf. [7, Proposition 3.5]). Let $R$ be a $k$-algebra and $A=R[D ; \sigma, \delta]$ be an Ore extension. Suppose that, for each finite-dimensional subspace $U$ of $R$, there exists a finite-dimensional subspace $V$ of $R$ such that $U \subseteq V, \sigma(V) \subseteq V$ and $\delta(V) \subseteq V^{p}$ for some $p \geqslant 1$. Then $\operatorname{GKdim}(A)=\operatorname{GKdim}(R)+1$.

We want to consider the Gelfand-Kirillov dimension of differential difference algebras satisfying 'similar' conditions as in Proposition 3.1. Our goal is to find a lower bound and an upper bound of the Gelfand-Kirillov dimension of such a differential difference algebra.

The following proposition gives a general lower bound of the Gelfand-Kirillov dimension of a differential difference algebra.

Proposition 3.2. Let $R$ be a $k$-algebra and $A=R[\mathcal{S}, \mathcal{D} ; \sigma, \delta]$ be a differential difference algebra of type $(m, n)$. Then $\operatorname{GKdim}(A) \geqslant \operatorname{GKdim}(R)+m+n$.

Proof. Suppose that $V$ is a finite-dimensional generating subspace of $R$ and $1 \in V$. Then

$$
W=V+\sum_{i=1}^{n} k D_{i}+\sum_{j=1}^{m} k S_{j}
$$

is a finite-dimensional generating subspace of $A$. For any $r \in \mathbb{N}$,

$$
W^{3 r}=\left(V+\sum_{i=1}^{n} k D_{i}+\sum_{j=1}^{m} k S_{j}\right)^{3 r} \supseteq \sum_{\substack{\alpha \in \mathbb{N}^{m}, \beta \in \mathbb{N}^{n} \\ 0 \leqslant|\alpha|,|\beta| \leqslant r}} V^{r} \mathcal{S}^{\alpha} \mathcal{D}^{\beta} .
$$

For any $k$-basis $U$ of $V^{r}$, the set

$$
\left\{u \mathcal{S}^{\alpha} \mathcal{D}^{\beta}: u \in U, 0 \leqslant|\alpha|,|\beta| \leqslant r, \alpha \in \mathbb{N}^{m}, \beta \in \mathbb{N}^{n}\right\}
$$

is a $k$-basis of $\sum_{0 \leqslant|\alpha|,|\beta| \leqslant r} V^{r} \mathcal{S}^{\alpha} \mathcal{D}^{\beta}$. Hence, we have that

$$
\begin{aligned}
\operatorname{dim}\left(W^{3 r}\right) \geqslant & \operatorname{dim}\left(\sum_{0 \leqslant|\alpha|,|\beta| \leqslant r} V^{r} \mathcal{S}^{\alpha} \mathcal{D}^{\beta}\right) \\
= & \operatorname{dim}\left(V^{r}\right) \cdot \operatorname{card}\left(\left\{\alpha: 0 \leqslant \alpha_{1}+\ldots+\alpha_{m} \leqslant r\right\}\right) \\
& \cdot \operatorname{card}\left(\left\{\beta: 0 \leqslant \beta_{1}+\ldots+\beta_{n} \leqslant r\right\}\right) \\
= & \operatorname{dim}\left(V^{r}\right) \cdot\left(\begin{array}{c}
r+m-1 \\
m
\end{array}\right) \cdot\left(\begin{array}{c}
r+n-1 \\
n
\end{array}\right),
\end{aligned}
$$

where $\left(\begin{array}{c}r+m-1 \\ m\end{array}\right)$ and $\left(\begin{array}{c}r+n-1 \\ n\end{array}\right)$ are polynomials in $r$ of degree $m$ and $n$ respectively. Hence,

$$
\begin{aligned}
\operatorname{GKdim}(A) & \geqslant \varlimsup_{r \rightarrow \infty} \log _{r} \operatorname{dim}\left(W^{r}\right)=\varlimsup_{r \rightarrow \infty} \log _{r} \operatorname{dim}\left(W^{3 r}\right) \\
& \geqslant \varlimsup_{r \rightarrow \infty} \log _{r}\left(\operatorname{dim}\left(V^{r}\right) \cdot\left(\begin{array}{c}
r+m-1 \\
m
\end{array}\right) \cdot\left(\begin{array}{c}
r+n-1 \\
n
\end{array}\right)\right) \\
& =\operatorname{GKdim}(R)+m+n .
\end{aligned}
$$


In the special case $R=k$, the equality in the above proposition holds, i.e. we have the following proposition, which indicates that the lower bound of $\operatorname{GKdim}(A)$ obtained in Proposition 3.2 cannot be sharpened any further.

Proposition 3.3. Let $A=k[\mathcal{S}, \mathcal{D} ; \sigma, \delta]$ be a differential difference algebra of type $(m, n)$ over $k$. Then $\operatorname{GKdim}(A)=m+n$.

Proof. Let $V=k+\sum_{i=1}^{n} k D_{i}+\sum_{j=1}^{m} k S_{j}$. Then $V$ is a finite-dimensional generating subspace of $A$. For any $r \in \mathbb{N}$,

$$
V^{r}=\left(k+\sum_{i=1}^{n} k D_{i}+\sum_{j=1}^{m} k S_{j}\right)^{r} \subseteq \sum_{0 \leqslant|\alpha|,|\beta| \leqslant r} k \mathcal{S}^{\alpha} \mathcal{D}^{\beta},
$$

where the last inclusion holds since

$$
\mathcal{D}^{\beta} \mathcal{S}^{\alpha} \in \sum_{\beta^{\prime} \in \mathbb{N}^{n},\left|\beta^{\prime}\right|=|\beta|} k \mathcal{S}^{\alpha} \mathcal{D}^{\beta^{\prime}}, \quad \alpha \in \mathbb{N}^{m}, \beta \in \mathbb{N}^{n} .
$$

So, $\operatorname{dim}\left(V^{r}\right) \leqslant\left(\begin{array}{c}r+m-1 \\ m\end{array}\right) \cdot\left(\begin{array}{c}r+n-1 \\ n\end{array}\right)$. Hence, $\operatorname{GKdim}(A) \leqslant m+n$ and thus by Proposition 3.2 $\operatorname{GKdim}(A)=m+n$.

Now let us turn to upper bounds for $\operatorname{GKdim}(A)$. First we consider the case when $R$ is finitely generated.

Lemma 3.4. Let $R$ be a $k$-algebra with a finite-dimensional generating subspace $V$, and let $A=R[\mathcal{S}, \mathcal{D} ; \sigma, \delta]$ be a differential difference algebra of type $(m, n)$. Suppose that $\sigma_{i}(V) \subseteq V$ for $1 \leqslant i \leqslant m$. Then $\operatorname{GKdim}(A) \leqslant 2 \operatorname{GKdim}(R)+m+n$.

Furthermore, if, for all $1 \leqslant i \leqslant m$ and $1 \leqslant j \leqslant n, \sigma_{i}\left(D_{j}\right)$ is contained in the vector space over $k$ generated by $\left\{D_{1}, \ldots, D_{n}\right\}$, then $\operatorname{GKdim}(A)=\operatorname{GKdim}(R)+m+n$.

Proof. Since $V$ is a generating subspace, there exists $p \geqslant 1$ such that

$$
a_{i j l} \in V^{p}, \quad \delta_{i}(V) \subseteq V^{p}, \quad 1 \leqslant i, l \leqslant n, 1 \leqslant j \leqslant m,
$$

where the $a_{i j l}$ are the coefficients that appear in Definition 2.1. Then

$$
\delta_{i}\left(V^{t}\right) \subseteq V^{p+t}, \quad \sigma_{j}\left(V^{t}\right) \subseteq V^{t}, \quad 1 \leqslant i \leqslant n, 1 \leqslant j \leqslant m, t \geqslant 1 .
$$

So, eventually replacing $V$ by $V^{p}$ if necessary, we may assume that

$$
1 \in V, \quad \delta_{i}(V) \subseteq V^{2}, \quad \sigma_{j}(V) \subseteq V, \quad 1 \leqslant i \leqslant n, 1 \leqslant j \leqslant m .
$$

Let $X=\sum_{i=1}^{n} k D_{i}, Y=\sum_{j=1}^{m} k S_{j}$ and $W=V+X+Y$. Then $W$ is a generating subspace of $A$.

In order to finish the proof of the first statement of the this lemma, we have to prove the following three lemmas first.

LEMma 3.5. For any integer $s \geqslant 1$,

(i) $X Y \subseteq V Y X, X V \subseteq V X+V^{2}, Y V=V Y$;

(ii) $X^{s} V \subseteq \sum_{i=0}^{s} V^{i+1} X^{s-i}$;

(iii) $X^{s} Y \subseteq \sum_{i=0}^{s-1} V^{s+i} Y X^{s-i}$;

(iv) if $\sigma_{i}\left(D_{j}\right) \in X$ for all $1 \leqslant i \leqslant m$ and $1 \leqslant j \leqslant n$, then $X Y=Y X$. 
Proof. (i) It follows easily by definition. (ii) (By induction on $s$.) If $s=1$, then we have $X V \subseteq V X+V^{2}$ by the commutation rules of differential difference algebras. Suppose that $X^{r} V \subseteq \sum_{i=0}^{r} V^{i+1} X^{r-i}$ for $1 \leqslant r \leqslant s$. Then

$$
\begin{aligned}
X^{s+1} V & \subseteq X^{s}\left(V X+V^{2}\right) \subseteq \sum_{i=0}^{s} V^{i+1} X^{s-i+1}+\sum_{i=0}^{s} V^{i+1} X^{s-i} V \\
& \subseteq \sum_{i=0}^{s} V^{i+1} X^{s-i+1}+\sum_{i=0}^{s} V^{i+1} \sum_{j=0}^{s-i} V^{j+1} X^{s-i-j} \\
& =\sum_{i=0}^{s} V^{i+1} X^{s-i+1}+\sum_{i=0}^{s} \sum_{j=0}^{s-i} V^{i+j+2} X^{s-i-j} \\
& =\sum_{i=0}^{s} V^{i+1} X^{s-i+1}+\sum_{i=0}^{s} \sum_{l=i+1}^{s+1} V^{l+1} X^{s-l+1} \quad(l:=i+j+1) \\
& =\sum_{i=0}^{s} V^{i+1} X^{s-i+1}+\sum_{l=1}^{s+1} V^{l+1} X^{s-l+1} \\
& =\sum_{i=0}^{s+1} V^{i+1} X^{s-i+1} .
\end{aligned}
$$

Thus, part (ii) holds for all $s \geqslant 1$.

(iii) (By induction on $s$.) If $s=1$, then we have $V^{1} Y X^{1} \supseteq X Y$ by (i), and thus part (iii) holds. Suppose that $X^{r} Y \subseteq \sum_{i=0}^{r-1} V^{r+i} Y X^{r-i}$ for all $1 \leqslant r \leqslant s$. Then,

$$
\begin{aligned}
X^{s+1} Y & \subseteq X^{s}(V Y X) \subseteq \sum_{i=0}^{s} V^{i+1} X^{s-i} Y X \quad \text { (by part (ii)) } \\
& =\sum_{i=0}^{s-1} V^{i+1} X^{s-i} Y X+V^{s+1} Y X \\
& \subseteq \sum_{i=0}^{s-1} V^{i+1} \sum_{j=0}^{s-i-1} V^{s-i+j} Y X^{s-i-j+1}+V^{s+1} Y X \\
& =\sum_{i=0}^{s-1} \sum_{j=0}^{s-i-1} V^{s+j+1} Y X^{s-i-j+1}+V^{s+1} Y X \\
& =\sum_{i=0}^{s-1} \sum_{l=i}^{s-1} V^{s+l-i+1} Y X^{s-l+1}+V^{s+1} Y X \quad(l:=i+j) \\
& \subseteq \sum_{i=0}^{s-1} \sum_{l=0}^{s-1} V^{s+l+1} Y X^{s-l+1}+V^{s+1} Y X \\
& =\sum_{l=0}^{s-1} V^{s+l+1} Y X^{s-l+1}+V^{s+1} Y X \subseteq \sum_{l=0}^{s} V^{s+l+1} Y X^{s-l+1}
\end{aligned}
$$

Hence, part (iii) holds.

(iv) This is straightforward.

Lemma 3.6. For all $r \geqslant 1, W^{r} \subseteq \sum_{i=0}^{r} \sum_{j=0}^{r-i} V^{2 r^{2}} Y^{i} X^{j}$. 
Proof. (By induction on $r$.) If $r=1$, then the right-hand side of the inclusion is $V^{2}+V^{2} X+$ $V^{2} Y \supseteq W$. Suppose the statement is true for $r \geqslant 1$. Then, by induction hypothesis,

$$
\begin{aligned}
& W^{r+1} \subseteq \sum_{i=0}^{r} \sum_{j=0}^{r-i} V^{2 r^{2}} Y^{i} X^{j}(V+X+Y) \quad \text { (induction hypothesis) } \\
& =\sum_{i=0}^{r} \sum_{j=0}^{r-i} V^{2 r^{2}} Y^{i} X^{j} V+\sum_{i=0}^{r} \sum_{j=0}^{r-i} V^{2 r^{2}} Y^{i} X^{j+1}+\sum_{i=0}^{r} \sum_{j=0}^{r-i} V^{2 r^{2}} Y^{i} X^{j} Y \\
& \subseteq \sum_{i=0}^{r} \sum_{j=0}^{r-i} V^{2 r^{2}} Y^{i} \sum_{l=0}^{j} V^{l+1} X^{j-l} \quad \text { (by Lemma 3.5(ii)) } \\
& +\sum_{i=0}^{r} \sum_{j=0}^{r-i} V^{2 r^{2}} Y^{i} X^{j+1} \\
& +\sum_{i=0}^{r} \sum_{j=0}^{r-i} V^{2 r^{2}} Y^{i} \sum_{l=0}^{j-1} V^{j+l} Y X^{j-l} \quad \text { (by Lemma 3.5(iii)) } \\
& =\sum_{i=0}^{r} \sum_{j=0}^{r-i} \sum_{p=0}^{j} V^{2 r^{2}+j-p+1} Y^{i} X^{p} \quad(p:=j-l) \\
& +\sum_{i=0}^{r} \sum_{j=1}^{r-i+1} V^{2 r^{2}} Y^{i} X^{j} \quad(\text { shift index } j) \\
& +\sum_{i=1}^{r+1} \sum_{j=0}^{r-i+1} \sum_{p=1}^{j} V^{2 r^{2}+2 j-p} Y^{i} X^{p} \quad(\text { shift } i \text { and } p:=j-l) \\
& \subseteq \sum_{i=0}^{r} \sum_{j=0}^{r-i} \sum_{p=0}^{r-i} V^{2 r^{2}+r-p+1} Y^{i} X^{p}+\sum_{i=0}^{r} \sum_{j=1}^{r-i+1} V^{2 r^{2}} Y^{i} X^{j} \\
& +\sum_{i=1}^{r+1} \sum_{j=0}^{r-i+1} \sum_{p=1}^{r-i+1} V^{2 r^{2}+2 r-p} Y^{i} X^{p} \\
& \subseteq \sum_{i=0}^{r} \sum_{p=0}^{r-i} V^{2 r^{2}+r-p+1} Y^{i} X^{p}+\sum_{i=0}^{r} \sum_{j=1}^{r-i+1} V^{2 r^{2}} Y^{i} X^{j}+\sum_{i=1}^{r+1} \sum_{p=1}^{r-i+1} V^{2 r^{2}+2 r-p} Y^{i} X^{p} \\
& \subseteq \sum_{i=0}^{r+1} \sum_{j=0}^{r-i+1} V^{2(r+1)^{2}} Y^{i} X^{j}
\end{aligned}
$$

Therefore, $W^{r} \subseteq \sum_{i=0}^{r} \sum_{j=0}^{r-i} V^{2 r^{2}} Y^{i} X^{j}$ for any $r \geqslant 1$.

Lemma 3.7. Let $f: \mathbb{N} \rightarrow \mathbb{R}$ be an increasing and positive valued function, and $p>1$. Then

$$
\varlimsup_{n \rightarrow \infty} \log _{n} f\left(p n^{2}\right) \leqslant 2 \varlimsup_{n \rightarrow \infty} \log _{n} f(n) .
$$

Proof. Let $d=\varlimsup_{n \rightarrow \infty} \log _{n} f(n)$. By [7, Lemma 2.1],

$$
d=\inf \left\{\rho \in \mathbb{R}: f(n) \leqslant n^{\rho} \text { for almost all } n \in \mathbb{N}\right\} .
$$

Hence, for any $\varepsilon>0, f(n)<n^{d+\varepsilon}$ for almost all $n$. So

$$
f\left(p n^{2}\right)<\left(p n^{2}\right)^{d+\varepsilon}=p^{d+\varepsilon} n^{2 d+2 \varepsilon}<n^{2 d+3 \varepsilon} \text { for almost all } n .
$$

Therefore, $\varlimsup_{\lim _{n \rightarrow \infty}} \log _{n} f\left(p n^{2}\right) \leqslant 2 d=2 \varlimsup_{\lim _{n \rightarrow \infty}} \log _{n} f(n)$. 
Now let us return to the proof of Lemma 3.4. Let $f(r)=\operatorname{dim}\left(V^{r}\right)$ for $r \in \mathbb{N}$. Then

$$
\begin{aligned}
\operatorname{GKdim}(A)= & \varlimsup_{r \rightarrow \infty} \log _{r} \operatorname{dim}\left(W^{r}\right) \leqslant \varlimsup_{r \rightarrow \infty} \log _{r} \operatorname{dim}\left(\sum_{i=0}^{r} \sum_{j=0}^{r-i} V^{2 r^{2}} Y^{i} X^{j}\right) \\
= & \varlimsup_{r \rightarrow \infty} \log _{r}\left(\operatorname{dim}\left(V^{2 r^{2}}\right) \sum_{i=0}^{r} \sum_{j=0}^{r-i} \operatorname{dim}\left(Y^{i}\right) \operatorname{dim}\left(X^{j}\right)\right) \\
= & \varlimsup_{r \rightarrow \infty} \log _{r}\left(f\left(2 r^{2}\right) \sum_{i=0}^{r} \sum_{j=0}^{r-i}\left(\begin{array}{c}
i+m-1 \\
m-1
\end{array}\right)\left(\begin{array}{c}
j+n-1 \\
n-1
\end{array}\right)\right) \\
\leqslant & \varlimsup_{r \rightarrow \infty} \log _{r} f\left(2 r^{2}\right)+\varlimsup_{r \rightarrow \infty} \log _{r} \sum_{i=0}^{r}\left(\begin{array}{c}
i+m-1 \\
m-1
\end{array}\right) \\
& +\varlimsup_{r \rightarrow \infty} \log _{r} \sum_{j=0}^{r}\left(\begin{array}{c}
j+n-1 \\
n-1
\end{array}\right) \\
\leqslant & 2 \mathrm{GK} \operatorname{dim}(R)+m+n
\end{aligned}
$$

where the last inequality holds because of Lemma 3.7 and the fact that if $p(i)$ is a polynomial in $i$ of degree $s$, then $\sum_{i=0}^{r} p(i)$ is a polynomial in $r$ of degree $s+1$.

For the second statement of Lemma 3.4, we need the following result.

LEMMA 3.8. Under the assumptions of the second statement of Lemma 3.4, we have that $W^{r} \subseteq \sum_{i=0}^{r} \sum_{j=0}^{r-i} V^{2 r-i-j} Y^{i} X^{j}$ for all $r \geqslant 1$.

Proof. Similarly to Lemma 3.6, this lemma can be proved by induction on $r$. It is easy to check that the inclusion is true for $r=1$. Suppose $W^{r} \subseteq \sum_{i=0}^{r} \sum_{j=0}^{r-i} V^{2 r-i-j} Y^{i} X^{j}$ for $r \geqslant 1$. Then, by the induction hypothesis and Lemma 3.5(ii) and (iv), we have that

$$
\begin{aligned}
W^{r+1} \subseteq & \sum_{i=0}^{r} \sum_{j=0}^{r-i}\left(V^{2 r-i-j} Y^{i} X^{j} V+V^{2 r-i-j} Y^{i} X^{j+1}+V^{2 r-i-j} Y^{i} X^{j} Y\right) \\
\subseteq & \sum_{i=0}^{r} \sum_{j=0}^{r-i} V^{2 r-i-j} Y^{i}\left(\sum_{l=0}^{j} V^{l+1} X^{j-l}\right) \quad \text { (by Lemma 3.5(ii)) } \\
& +\sum_{i=0}^{r} \sum_{j=0}^{r-i} V^{2 r-i-j} Y^{i} X^{j+1}+\sum_{i=0}^{r} \sum_{j=0}^{r-i} V^{2 r-i-j} Y^{i+1} X^{j} \\
= & \sum_{i=0}^{r} \sum_{j=0}^{r-i} \sum_{t=0}^{j} V^{2 r-i-t+1} Y^{i} X^{t} \quad(t:=j-l) \\
& \left.+\sum_{i=0}^{r} \sum_{j=1}^{r+1-i} V^{2 r-i-j+1} Y^{i} X^{j} \quad \text { (shift index } j\right) \\
& \left.+\sum_{i=1}^{r+1} \sum_{j=0}^{r-i} V^{2 r-i-j+1} Y^{i} X^{j} \quad \text { (shift index } i\right) \\
\subseteq & \sum_{i=0}^{r+1} \sum_{j=0}^{r+1-i} V^{2(r+1)-i-j} Y^{i} X^{j} .
\end{aligned}
$$

That proves our lemma. 
By the above lemma, $W^{r} \subseteq \sum_{i=0}^{r} \sum_{j=0}^{r-i} V^{2 r-i-j} Y^{i} X^{j} \subseteq \sum_{i=0}^{r} \sum_{j=0}^{r-i} V^{2 r} Y^{i} X^{j}$. Hence, by a similar argument as we used in the proof of the first statement of Lemma 3.4, we have that $\operatorname{GKdim}(A) \leqslant \operatorname{GKdim}(R)+m+n$. Thus, by Proposition 3.2, $\operatorname{GKdim}(A)=\operatorname{GKdim}(R)+m+n$.

Now we are in a position to state our main theorem.

Theorem 3.9. Let $R$ be a $k$-algebra and $A=R[\mathcal{S}, \mathcal{D} ; \sigma, \delta]$ be a differential difference algebra of type $(m, n)$. Suppose that for any finite-dimensional subspace $U$ of $R$ there exist a finitedimensional subspace $V$ of $R$ and an integer $p \geqslant 1$ such that

$$
U \subseteq V, \quad \sigma_{i}(V) \subseteq V, \quad \delta_{j}(V) \subseteq V^{p}, \quad 1 \leqslant i \leqslant m, 1 \leqslant j \leqslant n .
$$

Then

$$
\operatorname{GKdim}(R)+m+n \leqslant \operatorname{GKdim}(A) \leqslant 2 \operatorname{GKdim}(R)+m+n .
$$

Furthermore, if, for all $1 \leqslant i \leqslant m$ and $1 \leqslant j \leqslant n, \sigma_{i}\left(D_{j}\right)$ is contained in the vector space over $k$ generated by $\left\{D_{1}, \ldots, D_{n}\right\}$, then

$$
\operatorname{GKdim}(A)=\operatorname{GKdim}(R)+m+n .
$$

Proof. Let $W$ be a finite-dimensional subspace of $A$ with a $k$-basis $w_{1}, \ldots, w_{q}, q \in \mathbb{N}$. Note that each $w_{i}, 1 \leqslant i \leqslant q$, is a polynomial in $D_{1}, \ldots, D_{n}, S_{1}, \ldots, S_{m}$ with coefficients in $R$. Let $U$ be the subspace of $R$ spanned by all of the coefficients (in $R$ ) of $w_{1}, \ldots, w_{q}$ and all $a_{i j l}$ (defined in Definition 2.1), $1 \leqslant i, l \leqslant n, 1 \leqslant j \leqslant m$. Then $U$ is finite-dimensional and hence there exist a finite-dimensional subspace $V$ of $R$ and an integer $p \geqslant 1$ such that $U \subseteq$ $V, \sigma_{i}(V) \subseteq V, \delta_{j}(V) \subseteq V^{p}$ for $1 \leqslant i \leqslant m, 1 \leqslant j \leqslant n$. Let $B$ be the subalgebra of $R$ generated by $V$. Then $\sigma_{i}(B) \subseteq B, \sigma_{i}\left(D_{l}\right) \in B[\mathcal{D} ; \delta]$ and $\delta_{j}(B) \subseteq B$ for $1 \leqslant i \leqslant m, 1 \leqslant j, l \leqslant n$. That is, $A^{\prime}=B[\mathcal{S}, \mathcal{D} ; \sigma, \delta]$ is a differential difference algebra satisfying the conditions of Lemma 3.4. Note that $W \subseteq A^{\prime}$. So, by Lemma 3.4, we have

$$
\varlimsup_{r \rightarrow \infty} \log _{r} \operatorname{dim}\left(W^{r}\right) \leqslant \operatorname{GKdim}\left(A^{\prime}\right) \leqslant 2 \operatorname{GKdim}(B)+m+n \leqslant 2 \operatorname{GKdim}(R)+m+n .
$$

Thus $\operatorname{GKdim}(A) \leqslant 2 \operatorname{GKdim}(R)+m+n$ since $W$ is arbitrary. Therefore, by Lemma 3.2, $\operatorname{GKdim}(R)+m+n \leqslant \operatorname{GKdim}(A) \leqslant 2 \operatorname{GKdim}(R)+m+n$. That completes our proof of the first statement.

The second statement follows similarly by using the second part of Lemma 3.4.

Immediately from Theorem 3.9, we have the following corollaries.

Corollary 3.10. The quantum plane $k_{q}[x, y]$ has Gelfand-Kirillov dimension two.

Recall that an algebra $A$ is called locally finite-dimensional if every finitely generated subalgebra of $A$ is finite-dimensional.

Corollary 3.11. Let $R$ be a $k$-algebra and $A=R[\mathcal{S}, \mathcal{D} ; \sigma, \delta]$ be a differential difference algebra of type $(m, n)$ satisfying the conditions of the first statement of Theorem 3.9.

(i) If $R$ is locally finite-dimensional, then $\operatorname{GKdim}(A)=m+n$.

(ii) If $\operatorname{GKdim}(R)<\infty$, then $\operatorname{GKdim}(A)<\infty$.

Proof. (i) It follows from the fact that $\operatorname{GKdim}(R)=0$ if and only if $R$ is locally finitedimensional.

(ii) This is clear. 
Note that if we set $R=k$ in Theorem 3.9, then the conditions of the theorem are satisfied. Thus, Theorem 3.9 implies Proposition 3.3.

The following example shows that the upper bound of $\operatorname{GKdim}(A)$ stated in Theorem 3.9 is the 'best' one under the given conditions.

ExAmple 3.12. Let $A$ be the $k$-algebra generated by $\left\{z, z^{-1}, D, S\right\}$ with defining relations

$$
\begin{gathered}
\mathcal{R}=\left\{z z^{-1}=1, z^{-1} z=1, D z=z D, S z=z S,\right. \\
\left.D z^{-1}=z^{-1} D, S z^{-1}=z^{-1} S, D S=z S D\right\} .
\end{gathered}
$$

Let $R=k\left[z, z^{-1}\right]$ be the algebra of Laurent polynomials over $k$, and let $\sigma$ be the automorphism of the algebra $R[D] \subseteq A$ defined by

$$
\sigma\left(\sum_{i=0}^{l} c_{i} D^{i}\right)=\sum_{i=0}^{l} c_{i}(z D)^{i}, \quad l \geqslant 0, c_{i} \in R \text { for } 0 \leqslant i \leqslant l .
$$

Then $A=R[S, D ; \sigma, 0]$ is a differential difference algebra of type $(1,1)$ and

$$
\operatorname{GKdim}(A)=2 \operatorname{GKdim}(R)+1+1=4 .
$$

Proof. It is easy to see that $A$ can be thought of as an iterated Ore extension over $R$ : $A=R[S ;$ id, 0$]\left[D ; \sigma^{\prime}, 0\right]$ where $\sigma^{\prime}$ is the automorphism over $R[S]$ defined by

$$
\sigma^{\prime}\left(\sum_{i=0}^{l} c_{i} S^{i}\right)=\sum_{i=0}^{l} c_{i}(z S)^{i}, \quad l \geqslant 0, c_{i} \in R \text { for } 0 \leqslant i \leqslant l .
$$

Hence $\left\{S^{i} D^{j}: i, j \in \mathbb{N}\right\}$ forms an $R$-basis of $A$. Thus $A$ is a differential difference algebra.

Note that the restriction of $\sigma$ on $R$ is the identity automorphism of $R$. It is clear that $A$ satisfies all conditions of Theorem 3.9. So, by Theorem 3.9, $\operatorname{GKdim}(A) \leqslant 2 \operatorname{GKdim}(R)+2$. Since $\operatorname{GKdim}(R)=1$ (see, for example, [12, Corollary 8.2.15]), $\operatorname{GKdim}(A) \leqslant 4$.

Note that $D S=S \sigma(D)=S z D=z S D$. Then one can prove that $D^{j} S=z^{j} S D^{j}$ by induction on $j$, and then that $D^{j} S^{i}=z^{i j} S^{i} D^{j}$ by induction on $i$. Now we claim that

$$
B:=\left\{z^{l} S^{i} D^{j}: 0 \leqslant i+j \leqslant r, 0 \leqslant l \leqslant i j\right\} \subseteq W^{r}, \quad r \geqslant 1,
$$

where $W=k+k z+k z^{-1}+k D+k S$ is a generating subspace of $A$. Suppose $r \geqslant 1,0 \leqslant i+j \leqslant r$, $0 \leqslant l \leqslant i j$ and write $l=q j+p$ with $0 \leqslant q \leqslant i, 0 \leqslant p<j$. If $q=i$, then $p=0, l=i j$ and $z^{l} S^{i} D^{j}=z^{i j} S^{i} D^{j}=D^{j} S^{i} \in W^{r}$. If $q<i$, then

$$
\begin{aligned}
& S^{i-q-1} D^{p} S D^{j-p} S^{q}=z^{p} S^{i-q-1} S D^{p} D^{j-p} S^{q} \\
& \quad=z^{p} S^{i-q} D^{j} S^{q}=z^{p+q j} S^{i-q} S^{q} D^{j}=z^{l} S^{i} D^{j} .
\end{aligned}
$$

Since $(i-q-1)+p+1+(j-p)+q=i+j \leqslant r, z^{l} S^{i} D^{j}=S^{i-q-1} D^{p} S D^{j-p} S^{q} \in W^{r}$. Thus, our claim holds.

It is clear that $B$ is $k$-linearly independent. Then by our claim,

$$
\operatorname{dim}\left(W^{r}\right) \geqslant \operatorname{card}(B)=\sum_{i=0}^{r} \sum_{j=0}^{r}(i j+1)=\frac{1}{4} r^{4}+\frac{1}{2} r^{3}+\frac{5}{4} r^{2}+2 r+1 .
$$

Thus, $\operatorname{GKdim}(A) \geqslant \varlimsup_{r \rightarrow \infty} \log _{r} \operatorname{dim}\left(W^{r}\right) \geqslant 4$. Therefore, $\operatorname{GKdim}(A)=4$. 
Acknowledgements. The authors would like to thank the anonymous referee for his/her useful comments and suggestions. We also thank Professor Günter Krause for his careful reading of the manuscript and valuable comments.

\section{References}

1. A. D. Bell and K. R. Goodearl, 'Uniform rank over differential operator rings and Poincaré-BirkhoffWitt extensions', Pacific J. Math. 131 (1988) no. 1, 13-37.

2. N. Courtois, A. Klimov, J. Patarin and A. Shamir, 'Efficient algorithms for solving overdefined systems of multivariate polynomial equations', Advances in cryptology-EUROCRYPT 2000 (Springer, Berlin, 2000) 392-407.

3. C. HuH and C. O. KIM, 'Gelfand-Kirillov dimension of skew polynomial rings of automorphism type', Comm. Algebra 24 (1996) no. 7, 2317-2323.

4. P. E. Hydon, 'Symmetries and first integrals of ordinary difference equations', Proc. R. Soc. Lond. Ser. A 456 (2000) 2835-2855.

5. A. Kandri-Rody and V. Weispfenning, 'Non-commutative Gröbner bases in algebras of solvable type', J. Symbolic Comput. 9 (1990) no. 1, 1-26.

6. C. Kassel, Quantum groups, Graduate Texts in Mathematics 155 (Springer, Berlin, 1995).

7. G. Krause and T. Lenagan, Growth of algebras and Gelfand-Kirillov dimension, Graduate Studies in Mathematics 22 (American Mathematical Society, Providence, RI, 2000).

8. V. Levandovskyy and H. Schönemann, 'Plural: a computer algebra system for noncommutative polynomial algebras', Proceedings of the 2003 International Symposium on Symbolic and Algebraic Computation (ACM Press, New York, 2003) 176-183.

9. M. Lorenz, 'Gelfand-Kirillov dimension of skew polynomial rings', J. Algebra 77 (1982) no. 1, $186-188$.

10. E. L. MAnsfield and A. Szanto, 'Elimination theory for differential difference polynomials', Proceedings of the 2003 International Symposium on Symbolic and Algebraic Computation (ACM Press, New York, 2003) 191-198.

11. J. Matczuk, 'The Gelfand-Kirillov dimension of Poincaré-Birkhoff-Witt extensions', Perspectives in rings theory (eds F. Van Oystaeyen and L. Le Bruyn; Kluwer Academic Publishers, Dordrecht, 1988) 221-226.

12. J. C. McConnell, J. C. Robson and L. W. Small, Noncommutative Noetherian rings, Graduate Studies in Mathematics 30 (American Mathematical Society, Providence, RI, 2001).

13. J. J. ZhAng, 'A note on GK dimension of skew polynomial extensions', Proc. Amer. Math. Soc. 125 (1997) no. $2,363-374$.

Yang Zhang

Department of Mathematics

University of Manitoba

Winnipeg, $M B$

Canada R3T $2 \mathrm{~N} 2$

yang.zhang@umanitoba.ca

\author{
Xiangui Zhao \\ Department of Mathematics \\ University of Manitoba \\ Winnipeg, $M B$ \\ Canada R3T 2N2 \\ xian.zhao@umanitoba.ca
}

